\title{
Self-reported Quality of Life among Dental Students in Kingdom of Saudi Arabia
}

\author{
Salman A Alkallabi ${ }^{1}$, Navin A Ingle ${ }^{2}$, Mohammad A Baseer ${ }^{3}$, Jamal A Alsanea ${ }^{4}$, Sara A Alshammery $^{5}$, Asma S Almeslet ${ }^{6}$
}

\begin{abstract}
Introduction: Adverse physical and mental health conditions can affect the quality of life (QoL) and academic performance of undergraduate and postgraduate dental students and dental interns throughout their education.

Aim: To evaluate the QoL of undergraduate dental students, interns, and postgraduate dental students using the World Health Organization Quality of Life (WHOQOL)-BREF instrument in Kingdom of Saudi Arabia.

Materials and methods: A cross-sectional study was undertaken among undergraduate dental students, dental interns, and postgraduate dental students from various dental colleges of Kingdom of Saudi Arabia during the first semester of the academic year 2018-19. Information pertaining to the QoL was obtained by using a well-designed WHOQOL-BREF questionnaire, consisting of various domains of the QoL.

Results: A total of 518 dental students (UG $=273, \mathrm{DI}=135, \mathrm{PG}=110$ ) belonging to either gender ( $\mathrm{male}=196$ and female $=322$ ) from different academic years participated in this study. The overall Cronbach's a coefficient of the WHOQOL-BREF questionnaire was 0.793 . The highest mean score was found with the social relationships domain $(62.14 \pm 18.64)$, while physical health demonstrated the lowest mean score $(52.15 \pm 12.33)$. Dental students receiving government sponsorship for their education showed significantly higher mean scores for the psychological $(57.88 \pm 12.19$ vs $53.09 \pm 11.97, t=-4.498, p=0.000)$ and environmental $(62.93 \pm 14.54$ vs $57.94 \pm 13.46, t=-4.043, p=0.000)$ domains than the self-sponsored dental students. Similarly, government-sponsored dental students showed significantly higher QoL ( $3.93 \pm 0.75$ vs $3.52 \pm 0.91, t=-5.678, p=0.000)$ and satisfaction with health $(3.79 \pm 0.79$ vs $3.21 \pm 0.95, t=-7.509, p=0.000)$ compared to the selfsponsored dental students.

Conclusion: Dental interns demonstrated high overall QoL and satisfaction with health, while dental students receiving government funding for dental education showed higher scores for psychological health and environmental health domains. There is a need to improve the QoL of the students by considering various measures.
\end{abstract}

Keywords: Dental interns, Dental students, Postgraduate, Quality of life, Undergraduate, World Health Organization-Quality of life-BREF. Journal of Oral Health and Community Dentistry (2019): 10.5005/jp-journals-10062-0045

\section{INTRODUCTION}

Quality of life (QoL) is a multidimensional concept consisting of life satisfaction, emotional well-being, and functional characteristics of the individuals. According to the World Health Organization (WHO), QoL refers to the individuals' perception of their position in life in the context of the culture and value systems in which they live and in relation to their goals, expectations, standards, and concerns. ${ }^{1}$ Recently, health educators and researchers have focused more toward the QoL of health profession students. Studies reported that dental students are more likely to undergo higher levels of stress and tend to have more anxiety, depressive episodes, and burnout phenomenon compared to the laypeople. ${ }^{2-4}$

Several causes have been implicated in the increased stress level among the dental students. Female students tend to have more stress compared to the males. Staying away from the hometown, issues related to the manual dexterity and moving from the preclinical stage of dental education, and higher financial burdens were all found to be associated with increased stress levels among dental students. ${ }^{5,6} \mathrm{Al}$-Sowygh reported academic distress and perceived stress among Saudi dental students and reported higher levels of stress among the females, married, and those studying in higher academic years compared to their counterparts. In addition, Saleh et al. reported clinical training issues as the highest stressor among dental students. ${ }^{8}$

Stress and burnout affect adversely the QoL of dental students leading to educational difficulties. ${ }^{9,10}$ Prolonged years of dental

\footnotetext{
1,2Department of Dental Public Health, Riyadh Elm University, Riyadh, Kingdom of Saudi Arabia

${ }^{3,4}$ Department of Preventive Dentistry, Riyadh Elm University, Riyadh, Kingdom of Saudi Arabia

${ }^{5}$ Department of Restorative, Riyadh Elm University, Riyadh, Kingdom of Saudi Arabia

${ }^{6}$ Department of Oral Maxillofacial Surgery and Diagnostic Sciences, Riyadh Elm University, Riyadh, Kingdom of Saudi Arabia
}

Corresponding Author: Salman A Alkallabi, Department of Dental Public Health, Riyadh Elm University, Riyadh, Kingdom of Saudi Arabia, Phone: +966 548405299, e-mail: salman.a.alkallabi@student. riyadh.edu.sa

How to cite this article: Alkallabi SA, Ingle NA, et al. Self-reported Quality of Life among Dental Students in Kingdom of Saudi Arabia. J Oral Health Comm Dent 2019;13(2):44-49.

Source of support: Nil

Conflict of interest: None

education coupled with the tough and challenging nature of dental curricula may cause students to undergo remarkable levels of stress over the years. This can lead to burnout and behavioral and psychological problems impairing student's concentration abilities. These stressors can have bad sequelae on academic performance of the students, thereby producing negative impact on overall QoL. ${ }^{2,3}$

In Kingdom of Saudi Arabia, despite several published reports of stress among dental students, we did not find any literature on 
the overall QoL of dental students even after stress is one of the factors affecting the QoL. Evaluation of the dental students' QoL may provide important facts about their perception of life, health, and other vital factors.

Hence, this study aimed to evaluate the QoL of undergraduate dental students, interns, and postgraduate dental students using the World Health Organization Quality of life (WHOQOL)-BREF instrument in Kingdom of Saudi Arabia.

\section{Materials and Methods}

\section{Ethical Approval}

This research study was registered (FPGRP/43738005/279) with the research center of Riyadh Elm University, Riyadh, Kingdom of Saudi Arabia.

\section{Study Design, Settings, and Participants}

This cross-sectional study was conducted in both government and private dental colleges of Kingdom of Saudi Arabia. All the dental students (undergraduate dental students, dental interns, and postgraduate dental students) from different years of education were invited to participate in the study. Data were collected during the first semester of the academic year 2018-19 (SeptemberDecember).

\section{Questionnaire Contents}

The WHO (1998) developed the WHOQOL 100 and afterward the WHOQOL-BREF, a simplified version-to assess the QoL in different cultures and settings. ${ }^{11}$ The abbreviated WHOQOL-BREF has been reported similar reliability as that of longer 100 -item version. ${ }^{11,12}$ The WHOQOL-BREF questionnaire is made up of 26 items. Two items are stand-alone questions that evaluate overall QoL and satisfaction with health. The remaining 24 items are divided into four domains: (1) physical health (seven items), (2) psychological (six items), (3) social relationships (three items), and (4) environment (eight items). Every item is rated on a scale ranging from 1 to 5 , with domain scores scaled in a positive direction so that higher scores indicate a higher QoL. In our study, the demographic information section was also added along with other domains of the WHOQOLBREF (Table 1).

\section{Questionnaire Administration}

The WHOQOL-BREF questionnaire was digitalized by using the Google survey form, and the link was sent to the dental colleges' student's affair committee to be sent to all the students through registered e-mails. Moreover, the questionnaire link was also sent to the dental students' groups on social media in Kingdom of Saudi Arabia. All the efforts were made to exclude the personal

Table 1: WHOQOL-BREF domains and components

\begin{tabular}{|c|c|}
\hline Domain & Components \\
\hline \multirow[t]{6}{*}{ Personal information } & Gender \\
\hline & University \\
\hline & Level of dental education \\
\hline & Age \\
\hline & Marital status \\
\hline & Sponsor \\
\hline \multirow[t]{7}{*}{ Physical health } & Activities of daily living \\
\hline & Dependence on medicinal substances and medical aids \\
\hline & Energy and fatigue \\
\hline & Mobility \\
\hline & Pain and discomfort \\
\hline & Sleep and rest \\
\hline & Work capacity \\
\hline \multirow[t]{6}{*}{ Psychological } & Bodily image and appearance \\
\hline & Negative feelings \\
\hline & Positive feelings \\
\hline & Self-esteem \\
\hline & Spirituality/religion/personal beliefs \\
\hline & Thinking, learning, memory, and concentration \\
\hline \multirow[t]{3}{*}{ Social relationships } & Personal relationships \\
\hline & Social support \\
\hline & Sexual activity \\
\hline \multirow[t]{8}{*}{ Environment } & Financial resources \\
\hline & Freedom, physical safety, and security \\
\hline & Health and social care: accessibility and quality \\
\hline & Home environment \\
\hline & Opportunities for acquiring new information and skills \\
\hline & Participation in and opportunities for recreation/leisure activities \\
\hline & Physical environment (pollution/noise/traffic/climate) \\
\hline & Transport \\
\hline
\end{tabular}


identifiers of the participants' answers. Before completing the QoL questionnaire, students were asked to fill the personal information.

\section{Statistical Analysis}

Data obtained from the Google forms were downloaded in the Excel sheet and analysis performed. All the data were analyzed by using the statistical package for the social sciences (SPSS) version 25 (IBM Corp., Armonk, NY, USA). Descriptive statistics of frequency distribution, percentages, mean, and standard deviation values were calculated for the different domains of WHOQOL-BREF. Independent $t$ test was applied to compare the mean domain scores between different gender, marital status, and sponsor of the program, while the one-way analysis of variance test was applied to compare the various domain scores at the educational level of the study participants. A $p$ value of less than 0.05 was considered statistically significant for all statistical purposes.

\section{Results}

\section{Characteristics of the Participants}

A total of 518 subjects participated in the study. Higher number of females $(322 ; 62.2 \%)$ compared to the males $(196 ; 37.8 \%)$ responded to the questionnaire items. More than half $(273 ; 52.7 \%)$ of the participants were in undergraduate dental education while 135 (26.1\%) were interns, and 110 (21.2\%) were in postgraduate dental program. A very high number $(369 ; 71.2 \%)$ of participants were single, and more than half $(272 ; 52.2 \%)$ self-sponsored their fee of dental education. The mean age of the study participants was $24.90 \pm$ 4.77 years (Table 2 )

\section{WHOQOL-BREF Domains and Quality of Life and Satisfaction with Health Items}

The WHOQOL-BREF domains score for two questions on overall QoL and satisfaction with health were $(3.72 \pm 0.86)$ and (3.49 \pm $0.92)$ respectively. The responding students rated their overall QoL and satisfaction with their health as moderate. The highest mean score was found with the social relationships domain $(62.14 \pm$ 18.64), while physical health demonstrated the lowest mean score $(52.15 \pm 12.33)$ (Table 3$)$.

\section{WHOQOL-BREF Domain Scores and Educational Levels of the Participants}

When the WHOQOL-BREF domain scores were assessed among undergraduate dental students, dental interns, and postgraduate

Table 2: Characteristics of the study participants

\begin{tabular}{llll}
\hline Characteristics & & $n$ & $\%$ \\
\hline Gender & Male & 196 & 37.8 \\
& Female & 322 & 62.2 \\
& Total & 518 & 100.0 \\
Educational level & UG & 273 & 52.7 \\
& DI & 135 & 26.1 \\
& PG & 110 & 21.2 \\
Marital status & Total & 518 & 100.0 \\
& Single & 369 & 71.2 \\
& Married & 149 & 28.8 \\
Sponsor & Total & 518 & 100.0 \\
& Self-sponsored & 272 & 52.5 \\
& Government & 246 & 47.5 \\
Age (mean \pm SD) & Total & 518 & 100.0 \\
\hline
\end{tabular}

Table 3: WHOQOL-BREF domains and QoL and satisfaction with health items

\begin{tabular}{lrrll}
\hline Domain/item & Mean & \multicolumn{1}{l}{ SD } & Minimum & Maximum \\
\hline Physical health & 52.15 & 12.33 & 3.57 & 100.00 \\
Psychological health & 55.36 & 12.30 & 5.00 & 100.00 \\
Social relationships & 62.14 & 18.64 & 0.00 & 100.00 \\
Environment health & 60.31 & 14.19 & 3.13 & 100.00 \\
Overall QoL & 3.72 & 0.86 & 1.00 & 5.00 \\
Satisfaction with health & 3.49 & 0.92 & 1.00 & 5.00 \\
\hline
\end{tabular}

Scores on the four domains (physical health, psychological, social relationships, environment) could range from 0 to 100 ; scores on QoL and satisfaction with health scores ranged from 1 to 5 . Higher number indicates great quality and highest satisfaction for both scales.

dental students, physical health $(52.33,51.16$, and 52.93$)$ and psychological $(54.58,56.64$, and 55.71) domains showed lower mean scores compared to the social relationship (62.24, 60.59, and 63.79) and environment domains $(60.16,59.86$, and 61.21). The analysis of variance test did not show any statistically significant differences in mean physical health, psychological, social relationships, and environmental domains and educational level of the dental students $(p>0.05)$. The dental interns (3.87) showed highest overall QoL compared to the postgraduate (3.69) and undergraduate (3.65) dental students. This mean overall QoL score showed significant difference across different educational levels $(p<0.05)$. Similarly, dental interns demonstrated highest mean satisfaction score (3.62) compared to the postgraduate (3.55) and undergraduate (3.40) dental students. The mean satisfaction with health scores showed statistically significant difference across the different educational levels of the study participants $(p>0.05)$, as shown in Figure 1.

\section{WHOQOL-BREF Domain Scores between Genders}

In general, female dental students showed higher mean scores for physical health $(52.41 \pm 12.31$ vs $51.72 \pm 12.4, t=-0.62, p=0.535)$, psychological $(55.86 \pm 12.38$ vs $54.55 \pm 12.15, t=-1.177, p=0.24)$, social relationship $(62.64 \pm 19.03$ vs $61.31 \pm 18.01, t=-0.789$, $p=0.431)$, and environment domains $(61.04 \pm 13.95$ vs $59.11 \pm 14.54$ $t=-1.503, p=0.134$ ) compared to the male dental students with no significant differences $(p>0.05)$. Similarly, overall QoL $(3.74 \pm$ 0.87 vs $3.68 \pm 0.85, t=-0.681, p=0.496)$ and satisfaction with health $(3.5 \pm 0.93$ vs $3.46 \pm 0.91, t=-0.428, p=0.669)$ were found to be higher among females compared to the male dental students without any significant differences (Table 4).

\section{WHOQOL-BREF Domain Scores and Marital Status of the Dental Students}

The distribution of WHOQOL-BREF domain scores between single and married individuals is shown in Table 5, and there are no significant differences across physical health, psychological, social relationships, and environmental domains $(p>0.05)$. However, married dental students showed significantly higher QoL (3.87 \pm 0.85 vs $3.65 \pm 0.86, t=-2.65, p=0.009)$ and satisfaction with health $(3.7 \pm 0.83$ vs $3.4 \pm 0.95, t=-3.54, p=0.000)$ compared to the unmarried/single dental students.

\section{WHOQOL-BREF Domain Scores and Educational Sponsorship}

Dental students receiving government sponsorship for their education showed significantly higher mean scores for the 


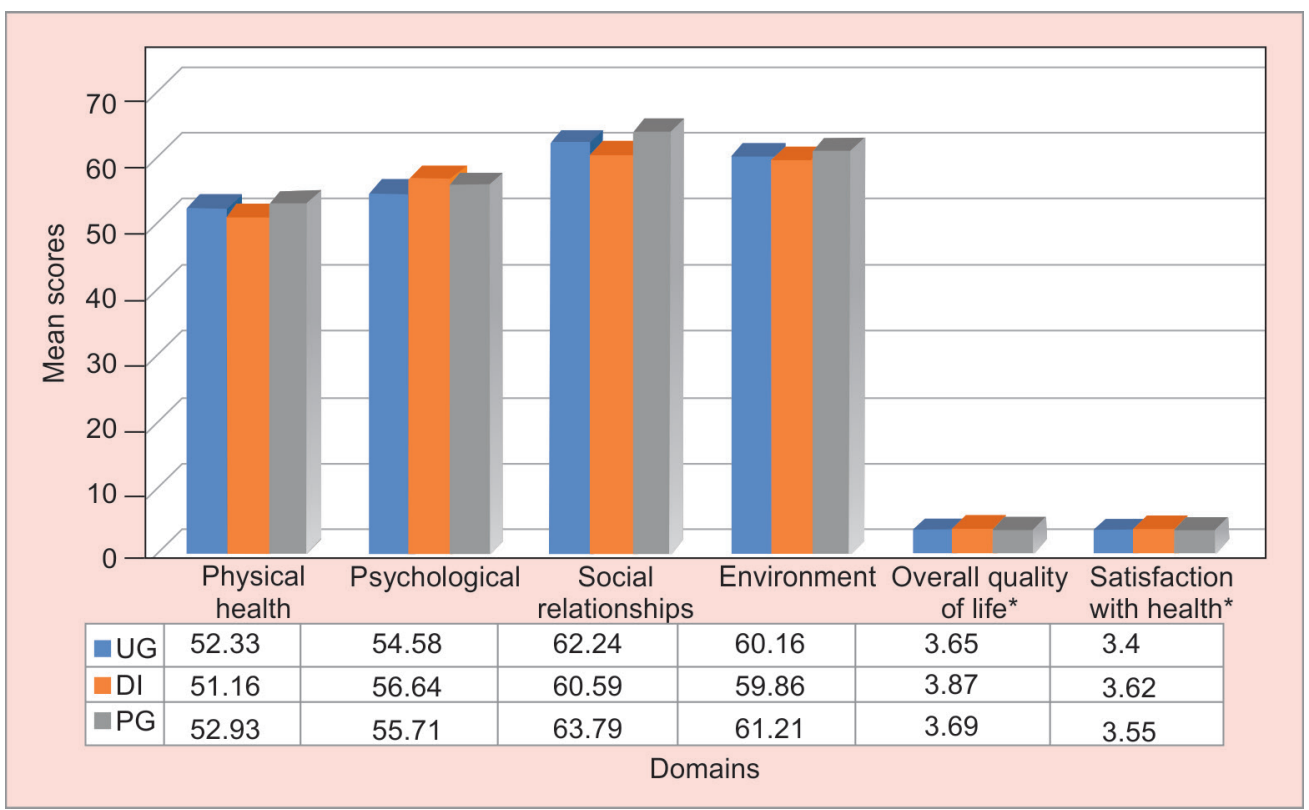

Fig. 1: Comparison of mean domain scores of WHOQOL-BREF in different educational levels $(*<0.05)($ UG, undergraduate dental students; DI, dental interns; PG, postgraduate dental students)

Table 4: Comparison of mean domain scores between different genders

\begin{tabular}{|c|c|c|c|c|c|c|}
\hline \multirow[b]{2}{*}{ Domain/item } & \multicolumn{2}{|c|}{ Male } & \multicolumn{2}{|c|}{ Female } & \multirow[b]{2}{*}{$t$} & \multirow[b]{2}{*}{$p$} \\
\hline & Mean & $S D$ & Mean & $S D$ & & \\
\hline Physical health & 51.72 & 12.4 & 52.41 & 12.31 & -0.62 & 0.535 \\
\hline Psychological & 54.55 & 12.15 & 55.86 & 12.38 & -1.177 & 0.24 \\
\hline Social relationships & 61.31 & 18.01 & 62.64 & 19.03 & -0.789 & 0.431 \\
\hline Environment & 59.11 & 14.54 & 61.04 & 13.95 & -1.503 & 0.134 \\
\hline Overall QoL & 3.68 & 0.85 & 3.74 & 0.87 & -0.681 & 0.496 \\
\hline Satisfaction with health & 3.46 & 0.91 & 3.5 & 0.93 & -0.428 & 0.669 \\
\hline
\end{tabular}

Table 5: Marital status and mean domain and QoL and satisfaction scores

\begin{tabular}{|c|c|c|c|c|c|c|}
\hline \multirow[b]{2}{*}{ Domain/Item } & \multicolumn{2}{|c|}{ Single } & \multicolumn{2}{|c|}{ Married } & \multirow[b]{2}{*}{$t$} & \multirow[b]{2}{*}{$p$} \\
\hline & Mean & $S D$ & Mean & $S D$ & & \\
\hline Physical health & 52.44 & 12.4 & 51.43 & 12.17 & 0.845 & 0.399 \\
\hline Psychological & 54.76 & 11.82 & 56.87 & 13.32 & -1.68 & 0.094 \\
\hline Social relationships & 61.66 & 18.39 & 63.32 & 19.26 & -0.911 & 0.363 \\
\hline Environment & 60.52 & 13.47 & 59.77 & 15.88 & 0.543 & 0.587 \\
\hline Overall QoL & 3.65 & 0.86 & 3.87 & 0.85 & -2.65 & 0.009 \\
\hline Satisfaction with health & 3.4 & 0.95 & 3.7 & 0.83 & -3.54 & 0.000 \\
\hline
\end{tabular}

Table 6: Educational sponsor and mean domain and QoL and satisfaction scores

\begin{tabular}{|c|c|c|c|c|c|c|}
\hline \multirow[b]{2}{*}{ Domain/item } & \multicolumn{2}{|c|}{ Self-sponsored } & \multicolumn{2}{|c|}{ Government } & \multirow[b]{2}{*}{$t$} & \multirow[b]{2}{*}{$p$} \\
\hline & Mean & $S D$ & Mean & $S D$ & & \\
\hline Physical health & 52.84 & 12.3 & 51.41 & 12.35 & 1.315 & 0.189 \\
\hline Psychological & 53.09 & 11.97 & 57.88 & 12.19 & -4.498 & 0.000 \\
\hline Social relationships & 60.98 & 19.46 & 63.41 & 17.65 & -1.486 & 0.138 \\
\hline Environment & 57.94 & 13.46 & 62.93 & 14.54 & -4.043 & 0.000 \\
\hline Overall QoL & 3.52 & 0.91 & 3.93 & 0.75 & -5.678 & 0.000 \\
\hline Satisfaction with health & 3.21 & 0.95 & 3.79 & 0.79 & -7.509 & 0.000 \\
\hline
\end{tabular}

psychological $(57.88 \pm 12.19$ vs $53.09 \pm 11.97, t=-4.498, \quad$ significantly higher QoL $(3.93 \pm 0.75$ vs $3.52 \pm 0.91, t=-5.678$, $p=0.000)$ and environmental $(62.93 \pm 14.54$ vs $57.94 \pm 13.46, \quad p=0.000)$ and satisfaction with health $(3.79 \pm 0.79$ vs $3.21 \pm 0.95$ $t=-4.043, p=0.000$ ) domains than the self-sponsored dental $t=-7.509, p=0.000$ ) compared to the self-sponsored dental students. Similarly, government-sponsored dental students showed students, as shown in Table 6. 


\section{Internal Consistency of the Items}

The internal consistency of the WHOQOL-BREF questionnaire items was calculated by the Cronbach's alpha coefficient. The overall Cronbach's alpha coefficient based on standardized items was found to be 0.793 .

\section{Discussion}

The present cross-sectional study was conducted among dental students (undergraduates, interns, postgraduates) to assess the QoL by using the WHOQOL-BREF protocol. In general, social relationships showed the highest score followed by environmental health, psychological, and physical health domains. This result suggests that social relationships and environmental health domains may have high impact of dental students' quality of rating rather than psychological and physical health domains. This finding is somewhat contradictory to the study reported by Andre et al., in which physical health and social relationships domains had greater impact on dental students' QoL. ${ }^{13}$

In this study, sociodemographic variables (gender, level of dental education, marital status, and sponsor for the education) were assessed to compare across the four WHOQOL-BREF domain scores and two stand-alone items. Gender, marital status, and level of education did not show any significant difference across four domains of WHOQOL-BREF. This finding is suggestive of the fact that sociodemographic factors did not contribute much for the variation in every domain score, as there are other factors that could easily influence the QoL of the students apart from the factors considered in this study. ${ }^{10,12,14}$ In our study, only scores of psychological and environmental health domains based on educational sponsor showed statistically significant $(p<0.05)$ differences. Governmentsponsored dental students showed significantly higher scores for the psychological and environmental health domains compared to the self-sponsored students for their dental education. This finding clearly suggests that dental school fee could have an impact on psychological and environmental health domains, as witnessed by lower scores among self-sponsored students.

Postgraduate students demonstrated higher scores in the physical health, psychological social relations, and environment domains as compared to undergraduate dental students and interns. This could be due to the larger number of years of clinical training, experience with the comprehensive patient care, and interaction with wider community compared to undergraduate dental students and interns. This finding is similar to the other studies in which years of dental education was considered as an important indicator of QoL among medical and dental students with the influence on psychological and social relationship domains. . $^{13,15}$

The physical health domain includes items like related energy, sleep, work capacity, and activities of daily living. Dental interns showed lower physical health domain, which is suggestive of the completion of the clinical requirements and fulfilment of the graduate competencies. In addition, internship training provides lesser requirements of clinical cases, didactic, and other challenges.

Psychological domain includes items that reflect the enjoyment of life, personal belief, concentration, bodily image, self-esteem, and negative feelings. In our study, undergraduate dental students showed lowest psychological score suggesting high work load, expectations, and stress experience during the academic years. In Kingdom of Saudi Arabia, dentistry program starts after two years of preparatory health sciences program. In the first two years of preclinical dental training program, students are evaluated on their performance based on competencies through simulation-based laboratory examinations. Later years of the dental program mainly focus on clinical training of the students, and the competencies are assessed mainly through patient care procedures along with comprehensive treatments and case presentations. Capstone courses have been identified within the program to measure the overall outcomes of the program. Halboub et al. reported that as the student progresses through the dental undergraduate curriculum, the level of stress increases accordingly. ${ }^{16}$ This could be one of the factors resulting in lower psychological scores among undergraduate dental students. Similar finding was reported by Mahmoud and Fareed among medical students in which decreased QoL was observed needing psychological support. ${ }^{17}$

The dental interns showed significantly higher scores for the QoL and satisfaction with health. This could be due to the less workload and stress observed during the internship training program compared to the undergraduate or postgraduate students.

When comparison was made between male and female dental students with regards to physical health, psychological health, social relationships, and environmental health domains, females exhibited higher scores than the male counterparts. This could be attributed to various factors like emotional expression, sensitivity, and deeper social connection. ${ }^{18}$ However, no significant difference was observed in the scores across all the domains between male and female dental students.

Moreover, the psychological and social relationship domain scores were lower for those students who were single than for those who were married. This could be attributed to the continuous support from the spouse and others, influencing psychological, social relation, and environmental health. ${ }^{19}$ However, QoL domains did not show any significant differences between single and married dental students. Study by Henning et al. showed the relationship between marriage and lower psychological distress among medical students. ${ }^{20}$ While married male had lowest stress scores and unmarried females had highest stress levels, ${ }^{21}$ overall QoL and satisfaction with health were significantly higher among married dental students compared to the single dental students.

Unlike other studies, our study also has some limitations. Although study link was sent to many dental schools in Kingdom of Saudi Arabia, it was difficult to assess how many respondents belonged to the private and government dental schools. Further, the questionnaire did not capture the years of dental education, economic condition, social status, residence, and other variables from both undergraduate and postgraduate students. Crosssectional nature of the study indicates responses collected at one point of time. The survey questionnaire was distributed during the end of the semester, which could have influenced results as most of the study participants nearly completed the courses. There is likelihood that the respondents would have given favorable responses due to the social desirability leading to the biased information. Only few sociodemographic information was considered in our study.

Further studies are needed to examine the WHOQOL-BREF domain scores of dental students, dental practitioners, and other health professionals to assess the QoL.

\section{Conclusion}

Social relationship was the highly scored domain by the dental students, dental interns, and postgraduate students. Dental interns 
demonstrated higher overall QoL and satisfaction with health. In addition, dental students receiving government funding for their education showed higher scores for psychological health and environmental health domains. There is a need to improve the QoL of the students by considering various measures.

\section{References}

1. World Health Organization.. Division of Mental Health and Prevention of Substance Abuse. WHOQOL: measuring quality of life. Geneva: World Health Organization; 1997. Available from: http://www.who. int/iris/handle/10665/63482.

2. Alzahem AM, Van der Molen HT, et al. Stress management in dental students: a systematic review. Adv Med Educ Pract 2014;5:167-176. DOI: 10.2147/AMEP.S46211.

3. Elani HW, Allison PJ, et al. A systematic review of stress in dental students. J Dent Educ 2014;78(2):226-242.

4. Basudan S, Binanzan N, et al. Depression, anxiety and stress in dental students. Int J Med Educ 2017;8:179-186. DOI: 10.5116/ijme. 5910.b961.

5. Hayes A, Hoover JN, et al. Perceived causes of stress among a group of western Canadian dental students. BMC Res Notes 2017;10(1):714. DOI: 10.1186/s13104-017-2979-9.

6. Alhajj MN, Khader Y, et al. Perceived sources of stress amongst dental students: a multicountry study. Eur J Dent Educ Off J Assoc Dent Educ Eur 2018;22(4):258-271. DOI: 10.1111/eje. 12350.

7. Al-Sowygh ZH. Academic distress, perceived stress and coping strategies among dental students in Saudi Arabia. Saudi Dent J 2013;25(3):97-105. DOI: 10.1016/j.sdentj.2013.05.002.

8. Al-Saleh SA, Al-Madi EM, et al. Survey of perceived stressinducing problems among dental students, Saudi Arabia. Saudi Dent J 2010;22(2):83-88. DOI: 10.1016/j.sdentj.2010.02. 007.

9. Gorter RC, Freeman R. Burnout and engagement in relation with job demands and resources among dental staff in Northern Ireland. Community Dent Oral Epidemiol 2011;39(1):87-95. DOI: 10.1111/j.1600-0528.2010.00568.x.

10. Jurkat $H, H o ̈ f e r ~ S$, et al. [Quality of life, stress management and health promotion in medical and dental students. a comparative study].
Dtsch Med Wochenschr 1946 2011;136(23):1245-1250. DOI: 10.1055/s0031-1280544.

11. Skevington SM, Gunson KS, et al. Introducing the WHOQOL-SRPB BREF: developing a short-form instrument for assessing spiritual, religious and personal beliefs within quality of life. Qual Life Res Int J Qual Life Asp Treat Care Rehabil 2013;22(5):1073-1083. DOI: 10.1007/ s11136-012-0237-0.

12. Krägeloh CU, Kersten $P$, et al. Validation of the WHOQOL-BREF quality of life questionnaire for general use in New Zealand: confirmatory factor analysis and Rasch analysis. Qual Life Res Int J Qual Life Asp Treat Care Rehabil 2013;22(6):1451-1457. DOI: 10.1007/s11136-0120265-9.

13. Andre A, Pierre GC, et al. Quality of Life Among Dental Students: A Survey Study. J Dent Educ 2017;81(10):1164-1170. DOI: 10.21815/ JDE.017.074.

14. Jamali A, Tofangchiha S, et al. Medical students' health-related quality of life: roles of social and behavioural factors. Med Educ 2013;47(10):1001-1012. DOI: 10.1111/medu.12247.

15. Zhang Y, Qu B, et al. Quality of life of medical students in China: a study using the WHOQOL-BREF. PloS One 2012;7(11):e49714. DOI: 10.1371/journal.pone.0049714.

16. Halboub E, Alhajj MN, et al. Perceived Stress among Undergraduate Dental Students in Relation to Gender, Clinical Training and Academic Performance. Acta Stomatol Croat 2018;52(1):37-45. DOI: 10.15644/ asc52/1/6.

17. Mahmoud MA, Fareed M. Assessment of Quality of Life among Medical Students in Saudi Arabia: A Study Based on WHO-QOL-BREF Protocol. Int J Med Res Health Sci 2018;7(10):1-11.

18. Hannah A, Lim BT, et al. Emotional intelligence and clinical interview performance of dental students. J Dent Edu 2017;81(10):1164-1170. DOI: 10.21815/JDE.017.074.

19. Rakizadeh E, Hafezi F. Sense of Coherence as a Predictor of Quality of Life Among Iranian Students Living in Ahvaz. Oman Med 2015;30(6):447-454. DOI: 10.5001/omj.2015.88.

20. Henning K, Ey S, et al. Perfectionism, the imposter phenomenon and psychological adjustment in medical, dental, nursing and pharmacy students. Med Educ 1998;32(5):456-464. DOI: 10.1046/j.13652923.1998.00234.x.

21. Musser LA, Lloyd C. The relationship of marital status and living arrangement to stress among dental students. J Dent Educ 1985;49(8):573-578. 